\title{
Resin Permeation into Acid-conditioned, Moist, and Dry Dentin: A Paradigm using Water-free Adhesive Primers
}

\author{
F.R. Tay, A.J. Gwinnett" ${ }^{2 *}$, K.M. Pang ${ }^{1}$, and S.H.Y. Wei \\ Department of Children's Dentistry and Orthodontics and ${ }^{1}$ Oral Biology Unit, Faculty of Dentistry, The University of Hong Kong, 34 \\ Hospital Road, Hong Kong; ${ }^{2}$ Department of Oral Biology and Pathology, School of Dental Medicine, State University of New York at Stony \\ Brook, Long Island, New York 11794-8702, USA; * to whom correspondence should be addressed
}

\begin{abstract}
Preservation of the morphological integrity of demineralized dentin collagen in its hydrated state may account for the success observed in wet-bonding procedures. This study investigated the micromorphological differences between moist- and dry-bonding techniques with the use of: (a) Aelitebond, an alcohol-based, water-free, single-component dentin adhesive primer system; and (b) a water-free, acetone-based experimental primer similar to the acetone-based, water-containing All-Bond 2, a twocomponent primer system. In the wet groups, acidconditioned dentin surfaces were blotted so that they remained visibly moist prior to bonding. In the dry groups, dentin surfaces were air-dried for $30 \mathrm{sec}$. Following the bonding procedures, dentin discs in each group were laminated together by means of a chemical-cure resin and processed for scanning electron microscope (SEM) and transmission electron microscope (TEM) examination. Conditioning with $10 \% \mathrm{H}_{3} \mathrm{PO}_{4}$ for 20 sec. produced complete demineralization of the outer dentin. In the wet groups, banded collagen and interfibrillar spaces could be observed at the surface of the acid-conditioned dentin. Complete wetting of the loosely arranged collagen fibrils by the resin resulted in the formation of a hybrid layer. In the dry groups, only a very thin hybrid layer was observed on the dentin surface, along the walls of the tubules, and along the course of their lateral branches. The absence of banded collagen and interfibrillar spaces within these areas suggested the existence of a collapsed dentin matrix along various liquid-vapor boundaries that restricted resin permeation into the subsurface intertubular matrix, producing an incompletely infiltrated "hybridoid region".
\end{abstract}

Key words: acid-conditioned dentin, moist and dry bonding, resin permeation, hybridoid region.

Received February 20, 1995; Accepted December 27, 1995

\section{Introduction}

Many of the current generation of dentin adhesives depend on the permeation of hydrophilic resin into chemically conditioned dentin (Erickson, 1992; Van Meerbeek et al., 1992; Eick et al., 1993). The application of acid conditioners removed the smear layer (Lee et al., 1973; Bowen, 1978; Berry et al., 1987) and depleted the outermost region of dentin of its inorganic phase (Chiba et al., 1989; Inokoshi et al., 1990; Ruse and Smith, 1991; Ikami et al., 1993; Tam and Pillar, 1994). Diffusion of resin into the demineralized, collagen-rich fibrous network resulted in "molecular entanglement of dental components" (Nakabayashi et al., 1995), producing a hybrid layer of resin-reinforced dentin (Nakabayashi, 1982). The existence of the hybrid layer in vivo has since been consistently demonstrated (Nakabayashi et al., 1992a, 1995; Gwinnett and Kanca, 1992a; Tay et al., 1994, 1995a; Walshaw and McComb, 1994).

Maintenance of the structural integrity of the collagen fibrils and micro-channels within the demineralized network facilitated optimal permeation of adhesive monomers (Nakabayashi et al., 1982). The presence of either denatured smear layer collagen remnants or denatured dentin surface collagen following acid application had been equated with a superficial, electron-dense zone within the hybrid layer that hindered resin permeation through the rest of the demineralized intertubular matrix (Van Meerbeek et al., 1993). Inclusion of ferric chloride in a citric acid conditioner (Mizunuma, 1986) or a salicyclic acid derivative in the primer (Hosoda et al., 1989) has been used as a chemical stabilizer of exposed collagen fibrils. Also along the line of chemical stabilization was the use of 2-hydroxyethyl methacrylate (HEMA) to reverse or re-expand a collapsed, demineralized dentin surface (Sugizaki, 1991), by improving the permeability of the bonding substrate (Nakabayashi and Takarada, 1992; Nakabayashi et al., 1992b; Watanabe et al., 1993; Mizuno et al., 1994).

Recently, improvement in bond strength was observed when some hydrophilic monomers were applied to moist dentin (Gwinnett, 1992; Kanca, 1992; Perdigao and Swift, 1994). The presence of moisture appeared to play a crucial 
role in preventing the demineralized surface collagen from collapsing following dehydration and accompanying surface tension stress (Gwinnett, 1994a). SEM studies of critical-point-dried specimens demonstrated that the physical integrity of the demineralized collagen could be preserved following the application of mild acids to dentin (Pashley et al., 1993; Titley et al., 1994). By means of an "environmental" SEM and cinematographic examination, it was found that the fibrous intertubular collagen network that initially accompanied moist, acid-conditioned dentin collapsed abruptly to form a smooth, featureless layer when the last trace of water evaporated from the dentin surface (Gwinnett, 1994b). However, remoistening dentin following a period of desiccation challenge of up to 30 seconds did not compromise the bond strength of an acetone-based adhesive to acid-conditioned dentin (Gwinnett, 1994a). To date, structural information on bonding to moist and dry dentin was all derived from SEM studies, and comparative ultrastructural data were lacking.

The purpose of this investigation was to compare the micromorphologic features of the resin-dentin interface when hydrophilic resin dissolved in acetone and/or ethanol was applied to acid-conditioned dentin under moist- and dry-bonding conditions. This was accomplished through an interactive study using SEM and TEM. To avoid the potential complication of creating a "pseudo-dry" condition resulting from re-wetting of briefly air-dried dentin (Gwinnett, 1994a) by the presence of water in some commercially available adhesive systems, we used a waterfree, alcohol-based system as well as a water-free, experimental version of a commercially available acetonebased system as models.

\section{Materials and methods}

Twenty-four erupted, non-carious, human third molars, stored in $0.5 \%$ chloramine $\mathrm{T}$, were used within one month following extraction. The roots were severed along the cemento-enamel junction by means of a low-speed machine equipped with a rotating diamond-impregnated copper disc under copious water supply, followed by the removal of occlusal enamel by means of a second parallel section. The bonding surface of the resulting dentin disc, from 1 to $1.5 \mathrm{~mm}$ thick, was then prepared with 600-grit silicon carbide sandpaper used to produce a flat surface devoid of enamel remnants or pulp exposures. Discs were randomly divided into four treatment groups, each containing six discs. The dentin discs were all conditioned with $10 \%$ phosphoric acid gel (All-etch semigel, Bisco, Itasca, IL) for $20 \mathrm{sec}$ and rinsed with distilled water for $20 \mathrm{sec}$.

Aelitebond (Bisco, Itasca, IL; batch \# 089417), an alcoholbased, water-free, single-component dentin adhesive primer system containing diarylsulfonedimethacrylate (DSDM) was used in the first two groups.

\section{DSDM moist group}

After the acid conditioner was rinsed off, excessive water on each dentin disc was blotted (Kanca technique) with a small piece of tissue paper (Kimwipes, Kimberly Clark Corporation,
Roswell, GA). Two coats of primer were immediately applied followed by gentle blowing with oil- and dust-free air (Falcon Safety Products, Inc., Branchburg, NJ) to cause the alcohol in the primer to evaporate. Bonding resin was then brushed onto each primed dentin disc, followed by light-curing for $20 \mathrm{sec}$ by means of a Max Lite (L.D. Caulk/Dentsply, Milford, DE) with the output intensity registered by a light meter (Cure Rite, L.D. Caulk/Dentsply, Milford, DE).

\section{DSDM dry group}

After the acid conditioner was rinsed off, each dentin disc was airdried in a stream of oil- and dust-free air, blown horizontally across the dentin surface for $30 \mathrm{sec}$. The application of adhesive primer and bonding resin was exactly the same as for the moist group.

An experimental, acetone-based, dual-cure, two-bottle primer system, being a modified version of the commercially available All-Bond 2 (Bisco, Itasca, IL), was used for comparison. The primer A ( $2 \%$ sodium salt of $\mathrm{N}$-p-tolylglycineglycidyl methacrylate) used in this study was devoid of the $17.4 \%$ water present in the commercial product. Except for the modified version of primer $A$, the remaining components used throughout the experiment were from a conventional All-Bond 2 kit (Batch \# 029154).

\section{Experimental formulation moist group}

After the acid conditioner was rinsed off, excessive water on the dentin surface was blotted with a small piece of tissue paper. Equal drops of experimental primers A and B (14\% biphenyl dimethacrylate) were dispensed and mixed for $5 \mathrm{sec}$. Multiple coats ( 8 to 10 ) were applied to each dentin disc until the primer in the well was used up. Each disc was gently air-dried with oiland dust-free air for $20 \mathrm{sec}$ so that the acetone and ethanol would evaporate completely. The primers were light-cured for $20 \mathrm{sec}$, at which time a shiny surface was noted. A thin layer of bonding resin was then applied and polymerized for an additional $20 \mathrm{sec}$.

\section{Experimental formulation dry group}

After the acid conditioner was rinsed off, each dentin disc was airdried for $30 \mathrm{sec}$ in the manner already described. The priming and bonding procedure was exactly the same as in the moist group.

The six treated dentin discs from each group were bonded together into 3 disc-pairs by means of a thin layer of chemicalcure bonding resin, similar to the sandwich technique described by Inokoshi et al. (1990). The bonded disc-pairs were sectioned perpendicular to the flat dentin surface into approximately equal halves by means of a diamond-impregnated copper disc under copious water supply. One half of each disc-pair was used for SEM and the other half for TEM examination.

Two strips, approximately $1.5 \mathrm{~mm}$ wide, from one half of each disc-pair were prepared for SEM. Each strip was attached to the stub of a specimen grinder (Model 623, Gatan Inc., Pleasanton, CA), and the bonded interface was polished under running water with silicon-carbide papers of, successively, 600, 1000 , and 1200 grit, followed by $1-\mu \mathrm{m}$-size polishing sheets (Imperial Polishing Film, 3M Microfinishing Systems, St. Paul, $\mathrm{MN})$. One mounted polished strip from each of the 3 disc-pairs 


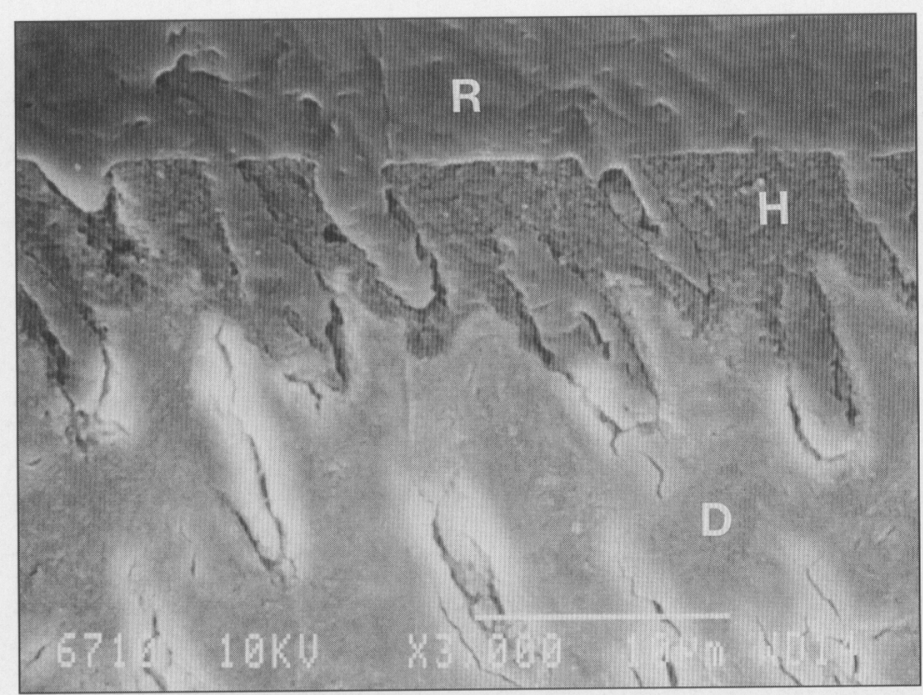

Figure 1a. Scanning electron micrograph of the resin-dentin interface in the DSDM moist group following plasma-etching. A hybrid layer $(\mathrm{H})$ of about $5 \mu \mathrm{m}$ was observed. Uneven removal of material along the interface, with the softer hybrid layer removed to a greater extent, resulted in a step between the resin layer (R) above and the intertubular dentin (D) below. Bar $=10 \mu \mathrm{m}$.

was plasma-etched with microwave-activated argon at $208 \mathrm{kPa}$ for $3 \mathrm{~min}$ under vacuum and water cooling (Plasma-Preen II, Model 862, Plasmatic Systems, Inc., North Brunswick, NJ), following a method reported by Hunter et al. (1993). Specimens were then ultrasonically cleaned in distilled water for $3 \mathrm{~min}$.

The other polished strip from each of the 3 disc-pairs in the same group was rinsed in $0.01 \mathrm{~N} \mathrm{HCl}$ for $20 \mathrm{sec}$. to bring the resin-dentin interface into relief through partial dissolution of the dentin. The prepared specimens were sputter-coated with gold in an ion sputtering machine (Fine coat, FC-1100, JEOL, Japan) at $1.2 \mathrm{kV}$ and $5 \mathrm{~mA}$ for 8 minutes. Observations were performed in a scanning electron microscope (JXA-840, JEOL, Japan) operated at 10 to $15 \mathrm{kV}$.

The other half of each disc-pair was completely demineralized in an aqueous solution of ethylene diamine tetraacetic acid (EDTA) buffered with sodium hydroxide to a $\mathrm{pH}$ of 7 , fixed in Karnovsky fixative for $4 \mathrm{hr}$, and rinsed with sodium cacodylate buffer. En bloc staining and post-fixation were performed simultaneously in a 1:1:1 by volume solution of: (a) $1 \%$ osmium tetroxide, (b) $0.1 \%$ ruthenium red, and (c) $0.2 \mathrm{M}$ sodium cacodylate buffer, $\mathrm{pH} 7.3$, at room temperature for $12 \mathrm{hr}$. Ruthenium red or its derivative as a negative stain has been shown to enhance the contrast of collagen (Luft, 1971; Garofalo et al., 1993). Specimens were further rinsed three times in cacodylate buffer, dehydrated in an ascending ethanol series (from $30 \%$ to $100 \%$ ), immersed in propylene oxide, and embedded in epoxy resin (TAAB 812 resin, TAAB Laboratories, UK). A 2-mm longitudinal strip was sectioned from the resin block, from which three $2 \times 2 \mathrm{~mm}$ blocks were trimmed and reembedded in epoxy resin to ensure proper orientation of the resin-dentin interface. Following examination of the semithin sections, $1 \mathrm{~mm} \times 1 \mathrm{~mm}$ ultrathin sections were prepared by means of a diamond knife and collected on 100-mesh copper grids (TAAB Laboratories, UK). The grids were double-stained with $2 \%$ uranyl acetate for $10 \mathrm{~min}$, followed by Reynolds' lead

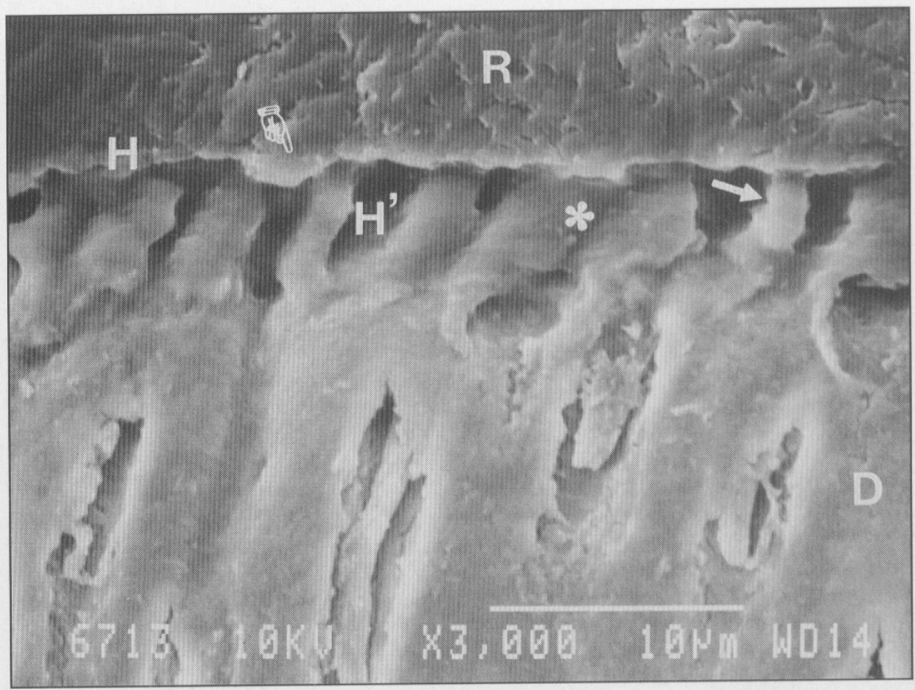

Figure 1b. Scanning electron micrograph of the resin-dentin interface in the DSDM dry group following plasma etching. A thin, surface hybrid layer $(\mathrm{H})$ immediately beneath the resin layer (R) could be observed together with part of a broken resin tag (pointer). In the "hybridoid region" $\left(\mathrm{H}^{\prime}\right)$ below, differential removal of the softer, partially infiltrated collagen (asterisk) resulted in an incomplete layer, where exposed resin tags (arrow) could be seen entering the underlying dentin (D). Bar $=10 \mu \mathrm{m}$.

citrate for an additional $5 \mathrm{~min}$. After being dried, they were examined with a transmission electron microscope (JEM-100, JEOL, Japan) operating at $80 \mathrm{kV}$.

\section{Results}

In the DSDM moist group, a hybrid layer of about $5 \mu \mathrm{m}$ could be observed in the SEM following plasma etching, with resin tags traversing from the resin layer above into the undemineralized dentin below (Fig. 1a). In the dry group, only a very thin hybrid layer was observed beneath the resin layer, with the underlying zone consisting of voids as well as individual resin tags (Fig. 1b). This incompletely infiltrated zone will be referred to as the "hybridoid region", to differentiate it from the thin, superficial hybrid layer. Material appeared to have been selectively removed, leaving behind resin tags as well as remnants of an amorphous material between the resin tags. The resin-dentin interface in the moist and dry groups, disclosed with acid dissolution, showed some features similar to those described above.

In the experimental formulation moist group, a hybrid layer of approximately $5 \mu \mathrm{m}$, brought into relief after acid dissolution, confirmed the SEM results disclosed by plasma etching (Fig. 2a). In the dry group, three different zones could be observed (Fig. 2b). A thin surface and basal hybrid layer was present, with a texture similar to that observed in the moist group. Lateral branches of the dentinal tubules were closely associated with the basal hybrid layer. The middle "hybridoid region" contained voids and incompletely infiltrated collagen that appeared collapsed and shrunken following air-drying of the specimens.

A TEM view of the resin-dentin interface in the DSDM moist group showed a hybrid layer that was clearly 


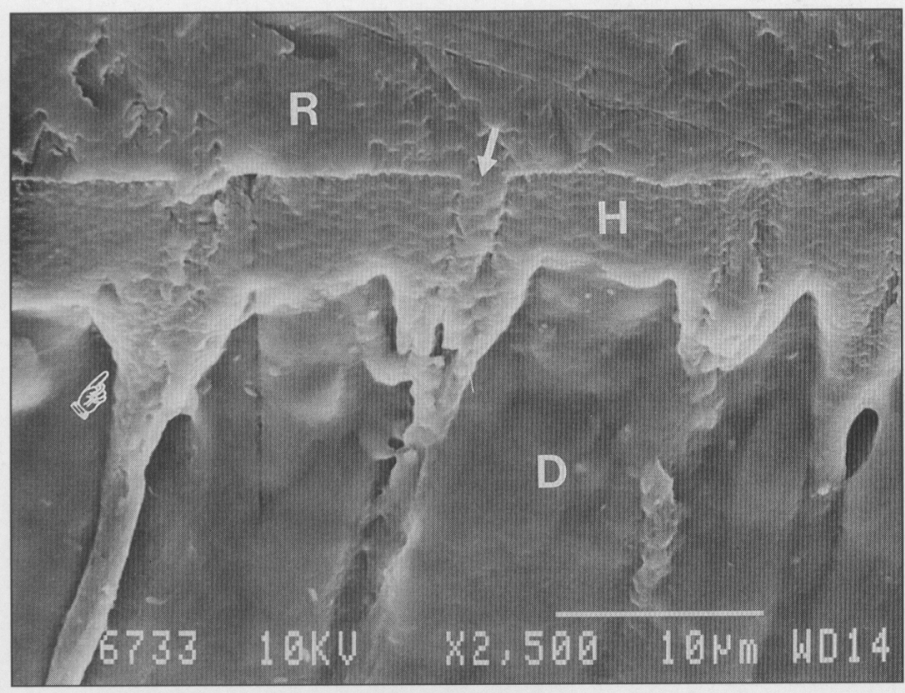

Figure 2a. Scanning electron image of the resin-dentin interface in the experimental formulation moist group that has been acid-rinsed. A distinct hybrid layer $(\mathrm{H})$ of about $5 \mu \mathrm{m}$ was observed. The resin layer (R) above could be seen extending as a resin tag (arrow) through the hybrid layer. A wedge-like extension of the hybrid layer formed a circumferential cuff around the periphery of the resin tag (pointer). The underlying dentin (D), being partially demineralized by acid-rinsing in $\mathrm{HCl}$ for SEM visualization, collapsed on air-drying and appeared at a lower level, revealing the resin tags in relief. Bar $=10 \mu \mathrm{m}$.

distinguishable from the underlying dentin (Fig. 3a) and consistent in its appearance from the surface to the base. Although a sharp demarcation existed between the base of

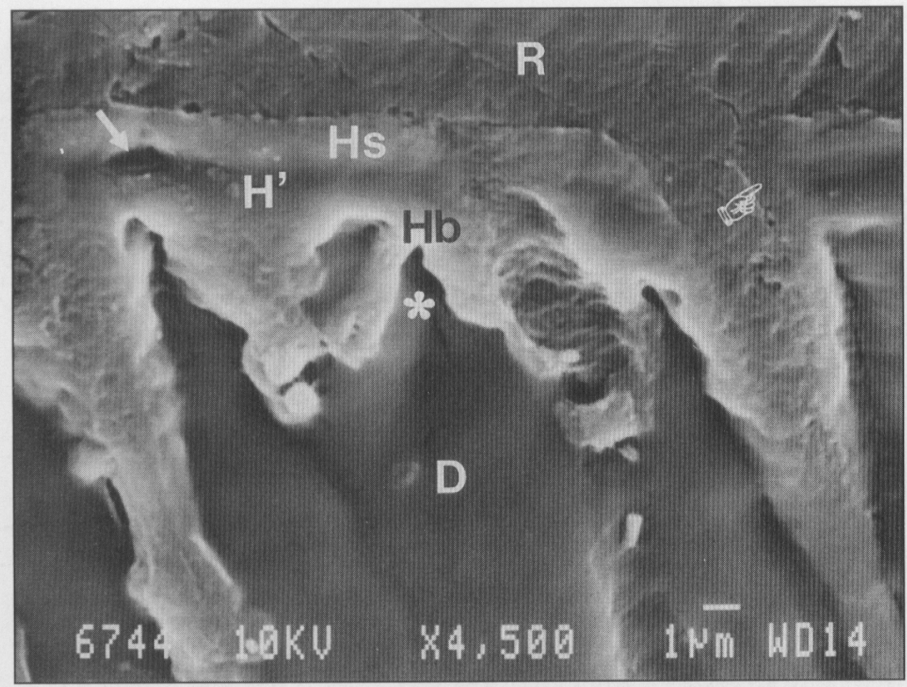

Figure $2 \mathrm{~b}$. Scanning electron micrograph of the resin-dentin interface in the experimental formulation dry group after acidrinsing, in which three zones could be distinguished: a surface (Hs) and a basal $(\mathrm{Hb})$ hybrid layer sandwiching a middle, collapsed "hybridoid region" $\left(\mathrm{H}^{\prime}\right)$. The surface hybrid layer represented the result of limited vertical intertubular monomer permeation through the acid-conditioned, surface intertubular dentin. The basal hybrid layer represented the contribution from dentinal tubule anastomosis (asterisk). The surface and basal hybrid layers were connected along the periphery of a dentinal tubule (pointer) through circumferential intertubular permeation from the tubule lumen. Arrow, void within "hybridoid region"; $\mathrm{R}$, resin layer; $\mathrm{D}$, dentin matrix demineralized by acid-rinse in $0.01 \mathrm{~N} \mathrm{HCl}$. Bar $=1 \mu \mathrm{m}$.

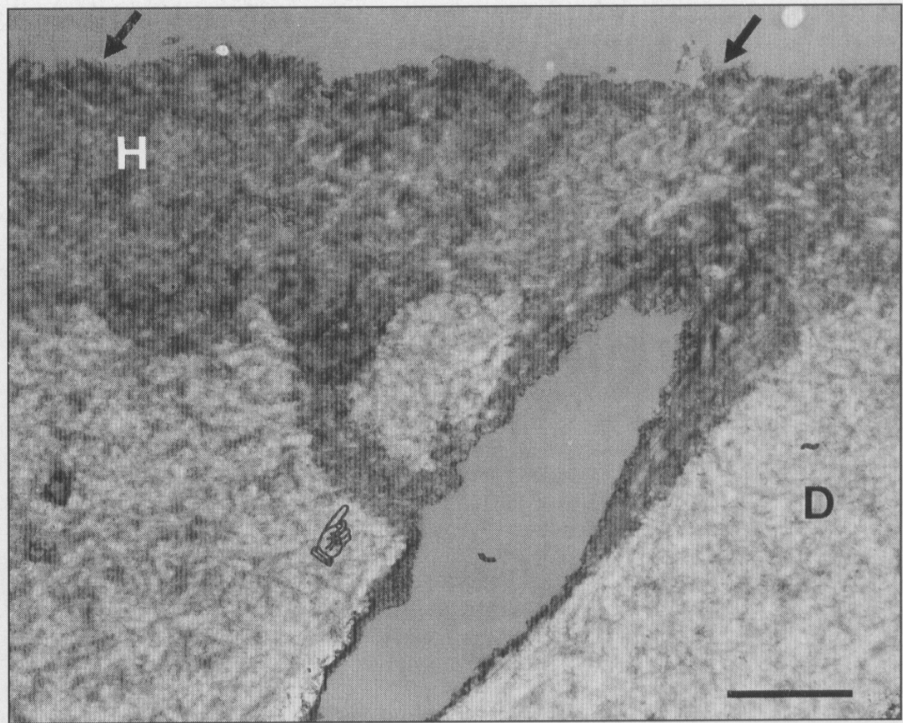

Figure 3a. Transmission electron micrograph of an overview of the resin-dentin interface in the DSDM moist group. Tiny protusions from the hybrid layer $(\mathrm{H})$ resulted in an uneven and rugged border (arrows). The basal portion of the hybrid layer (arrowheads), well demarcated from the underlying dentinal matrix (D) that was demineralized in EDTA for TEM preparation, was devoid of an electron-dense zone that was suggestive of the presence of encapsulated minerals. Pointer: contribution of resin infiltration through anastomosis of a lateral branch. Bar $=2 \mu \mathrm{m}$.

the hybrid layer and the underlying EDTA-demineralized dentinal matrix, there was no evidence of a partially demineralized zone of dentin at the base of the hybrid layer.

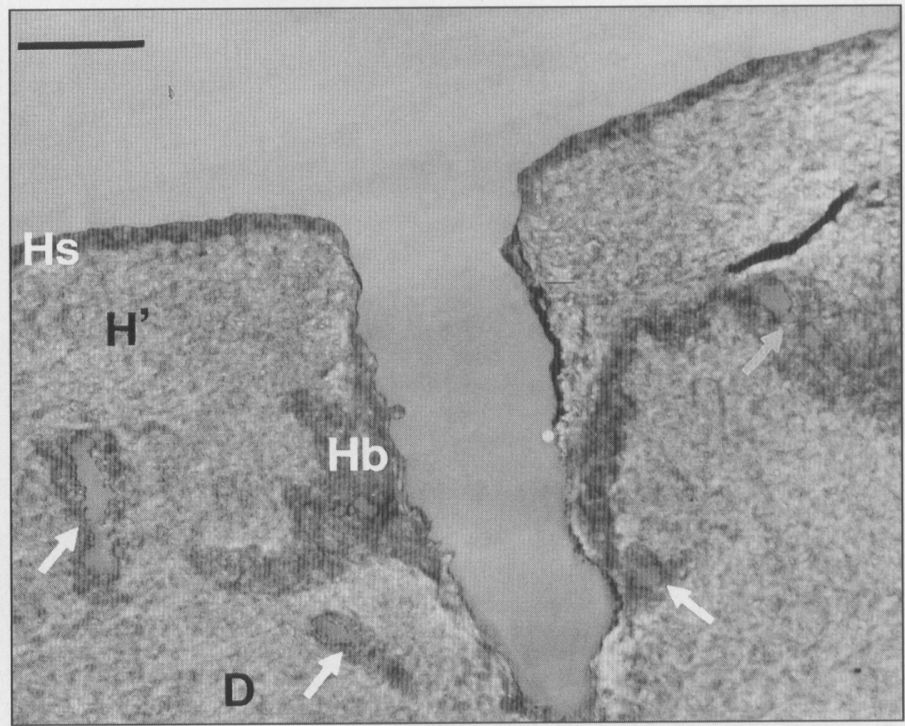

Figure $3 \mathbf{b}$. Transmission electron micrograph of an overview of the resin-dentin interface in the DSDM dry group. A thin, electrondense zone was observed along the dentin surface (Hs). The basal electron-dense zone $(\mathrm{Hb})$ was discontinuous and could be found circumferentially around the lateral branch of a dentinal tubule (arrows). In areas where discontinuity of the basal electron-dense zone occurred, there was no sharp demarcation between the lesselectron-dense "hybridoid region" $\left(\mathrm{H}^{\prime}\right)$ and the underlying dentinal matrix (D) that was demineralized in EDTA to facilitate TEM preparation. Bar $=2 \mu \mathrm{m}$. 


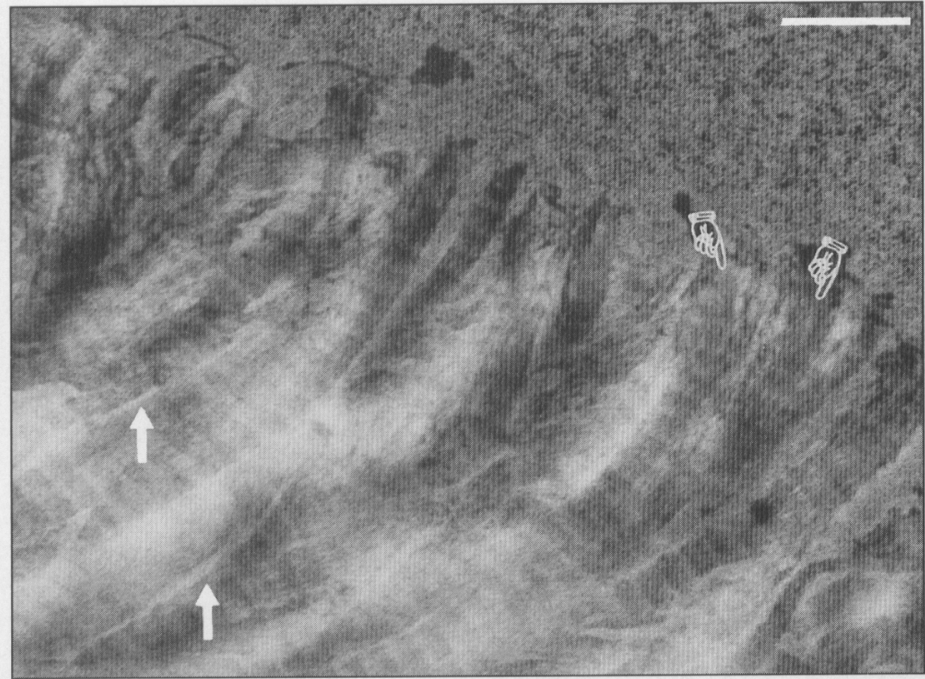

Figure 4a. Transmission electron micrograph of the surface of the hybrid layer in the DSDM moist group. Collagen fibrils could be traced to the surface of the hybrid layer, where they were arranged at right angles to the surface. Unraveling of microfibrils from the severed ends of some collagen fibrils could be observed (pointers). Interfibrillar channels (arrows) between adjacent collagen fibrils were evident. Bar $=250 \mathrm{~nm}$.

Air-drying the dentin surface for $30 \mathrm{sec}$ prior to the application of the dentin adhesive resulted in an incompletely infiltrated layer, wherein a "sandwich arrangement" could be distinguished, consisting of three zones (Fig. 3b). A thin electron-dense hybrid layer, similar to the staining characteristics of that in the moist group, was evident along the surface and basal portions, with a lesselectron-dense "hybridoid region" sandwiched in between. The basal hybrid layer, being roughly parallel to the surface hybrid layer, was discontinuous and with a tendency to form around the lateral branches of the dentinal tubules, which were filled with resin.

At higher magnification, the surface of the hybrid layer in the DSDM moist group consisted of banded collagen fibrils that could be traced at right angles to the dentin surface (Fig. 4a). Unraveling of individual microfibrils at the severed junction resulted in the appearance of loose strands, with the cross-striations from longitudinally sectioned collagen fibers clearly visible immediately underneath. Interfibrillar channels between adjacent collagen fibrils were evident. A view from a similar location in the DSDM dry group showed a surface zone where diffuse electron-dense patches were scattered within an area devoid of both collagen banding or interfibrillar spaces (Fig. 4b). Further down within the "hybridoid region", banding within collagen fibrils as well as interfibrillar spaces was evident. Similar features were observed along the basal portion as well as around the lateral branches (Fig. 4c).

An overall TEM view of a longitudinal section through a dentinal tubule in the experimental formulation moist group is shown in Fig. 5a. Within the hybrid layer, features similar to those observed in the DSDM moist group were present. In addition, remnants of the lamina limitans, seen as an electron-dense sheath, appeared within the resin tags. In a

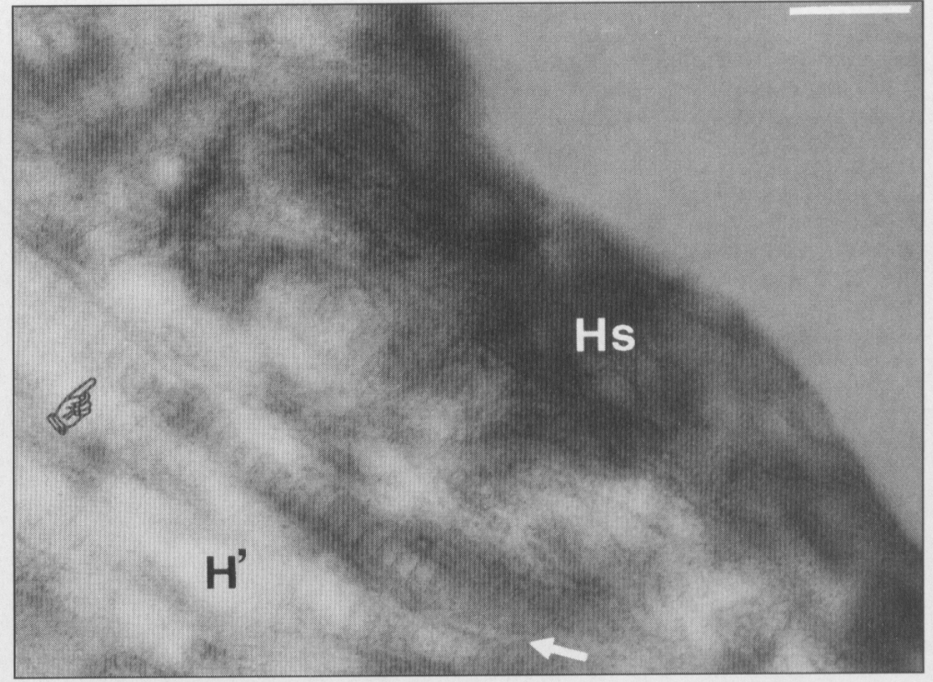

Figure $4 \mathrm{~b}$. A transmission electron micrograph of the dentin surface in the DSDM dry group. Collagen banding (pointer) or interfibrillar spaces (arrow), non-existent at the surface electron-dense zone (Hs), became more prominent within the sub-surface "hybridoid region" $\left(\mathrm{H}^{\prime}\right)$. Bar $=250 \mathrm{~nm}$.

comparable overall view of the experimental formulation dry group, there was a gradient of electron-density extending from the dentin surface into the subsurface "hybridoid region" (Fig. 5b). Electron-dense zones were found around the periphery of the dentinal tubules as well as their lateral branches, which were filled with resin.

In the moist groups, resin occupied initially the entire lumen at the orifice of the dentinal tubule. Further down, these short solid resin tags were replaced by resin globules, being attached to the periphery of the dentinal tubules via a thin resin sheath (Fig. 6a). In contrast, long solid resin tags could be observed in the dry groups (Fig. 6b). At higher magnification, diffusion of resin through the very thin, electron-dense lamina limitans resulted in the entrapment of a layer of amorphous material that appeared more like denatured protein remnants than partially dissolved hydroxyapatite crystallites (Fig. 6c).

\section{Discussion}

The dental literature is rich with scientific articles describing the interface between restorative materials and tooth tissue since Kramer and McLean published the first paper in 1952. Recent studies have added valuable information on the topographical or ultrastructural features of the resin-dentin interface, with the use of adhesive systems that compared different treatment philosophies of the smear layer and smear plugs (Nakabayashi, 1982, 1992; Abe, 1986; Takarada, 1990; Eick et al., 1991, 1992, 1993; Erickson, 1992; Van Meerbeek et al., 1992, 1993; Inokoshi et al., 1993; Watanabe et al., 1994). Reports on the structural aspects of the reaction of adhesive systems to moist- and dry-bonding conditions following the removal of the smear layer and the smear plugs with acid conditioners had been relatively scarce (Gwinnett and Kanca, 1992b; Titley et al., 1994).

In the present study, microstructural features of the water- 


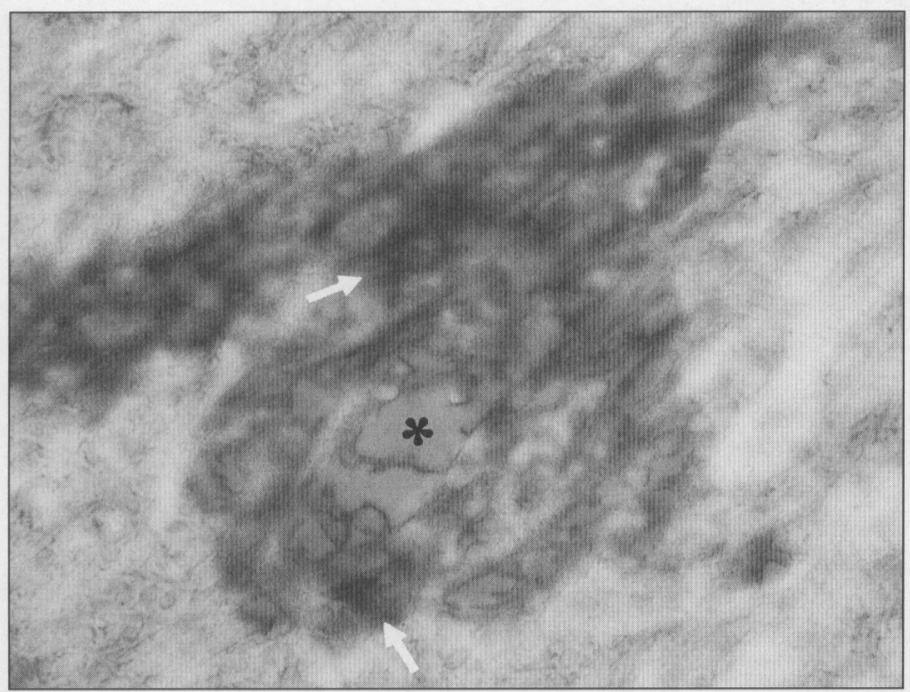

Figure 4c. A transmission electron micrograph of the basal electrondense zone in the DSDM dry group. A layer of electron-dense, resininfiltrated, partially denatured collagen (arrows) could be seen around the lateral branch of a dentinal tubule (asterisk). Bar $=500 \mathrm{~nm}$.

free resin systems were strikingly similar in their respective moist and dry groups. In contrast to the moist groups, the dry groups showed evidence of incomplete resin infiltration, with

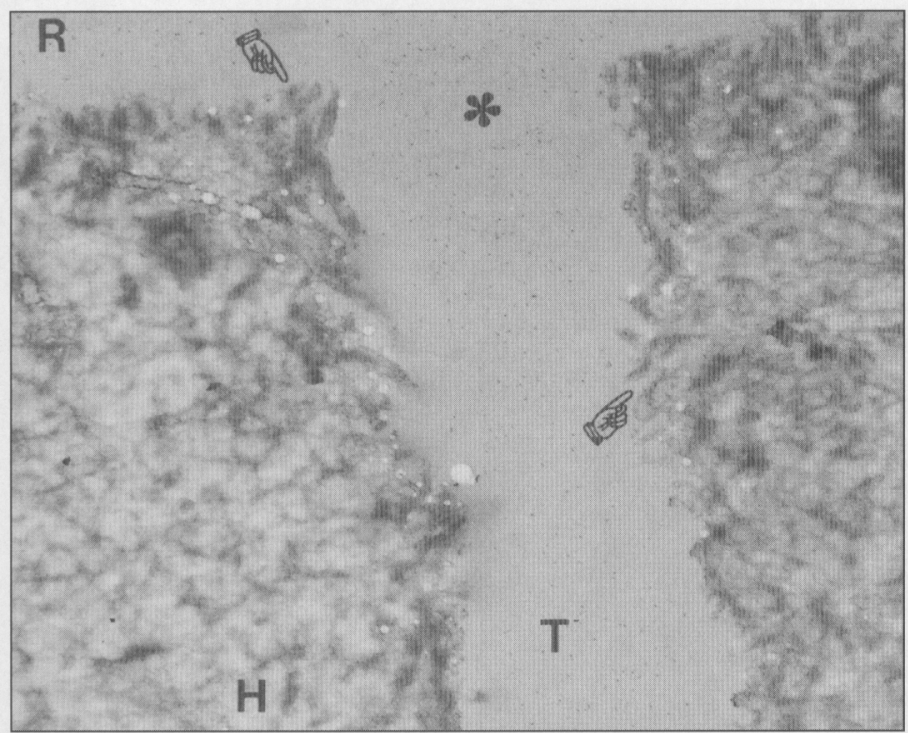

Figure 5a. Transmission electron micrograph of an overview of the resin-dentin interface from the experimental formulation moist group. The surface of the hybrid layer was devoid of any remnants of the smear layer, with the patency of the tubular orifice wellmaintained (asterisk). Collagen could be seen protruding from the surface of the intertubular dentin as well as from the walls of the tubular lumen (pointers). There appeared to be a uniform penetration of the adhesive resin throughout the hybrid layer $(\mathrm{H})$, with the absence of an electron-dense basal zone along the demineralization front (arrowheads). Remnants of the lamina limitans (arrows), the limiting membrane of the tubular lumen, could be seen partially detached from its peripheral location, resulting in a folded appearance further down the resin tag. $R$, resin layer; T, resin tag; D, underlying dentinal matrix that has been demineralized in EDTA for TEM preparation. Bar $=2 \mu \mathrm{m}$. the exposed, uninfiltrated collagen eroded to a greater extent during argon ion-etching (Miller et al., 1994), contributing to a so-called "hybridoid region". Using sodium hypochlorite to remove incompletely infiltrated collagen within the layer of resin impregnated dentin, researchers have previously reported a similar zone with a dry-bonding technique (Wang and Nakabayashi, 1991), or with a wet-bonding technique when dentin surfaces were conditioned with $65 \%$ phosphoric acid (Kato et al., 1994). Dentin conditioning by means of phosphoric acid with a concentration beyond $35 \%$ resulted in denaturation of the demineralized collagen (Mizunuma, 1986; Ikami et al., 1993; Reeve et al., 1994). Restricted resin permeation in the presence of denatured collagen (Pashley et al., 1993) could explain why only a very thin, surface hybrid layer was observed previously in a wet-bonding technique (Kato et al., 1994).

It was not clear from the SEM results why an additional basal zone could be observed below the "hybridoid region" in the dry groups. TEM results for the dry groups, however, indicated that the staining in the "hybridoid region" resembled that of the dentin matrix underneath that had been demineralized in EDTA for TEM preparation. The basal zone, on the other hand, had staining similar to that of the surface zone, and was often associated with resin-filled lateral branches extending from adjacent dentinal tubules. Thus, certain sites within and along the basal portion of the "hybridoid region" could be better infiltrated if they were

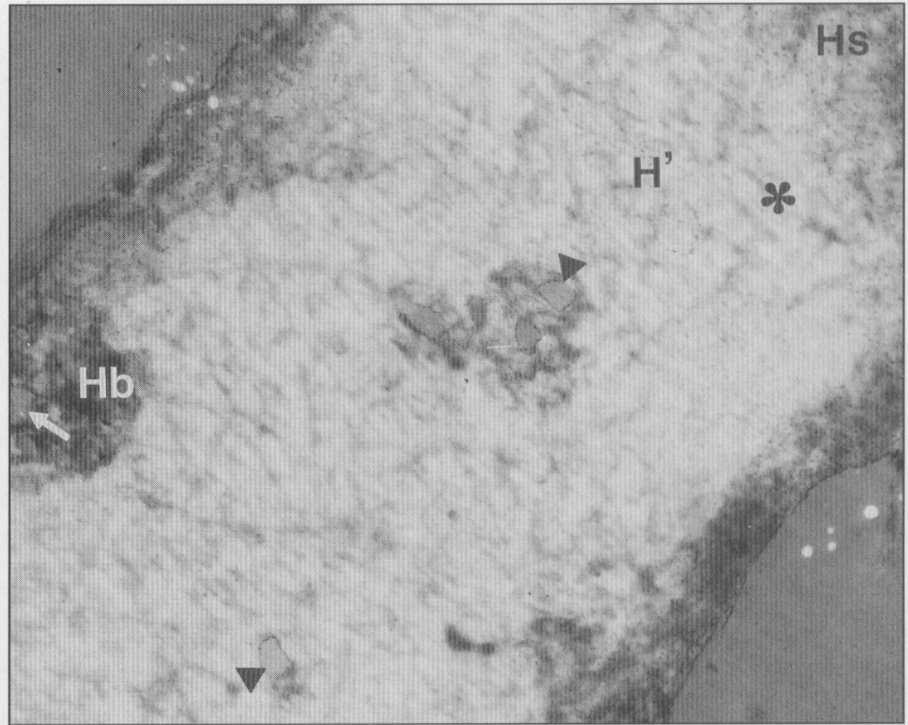

Figure 5b. Transmission electron micrograph from a specimen in the experimental dry group. The tubular orifice, being constricted (arrows) and obscured by some amorphous material (white asterisk), probably represented an artifact resulting from an oblique section through the curvature of a dentinal tubule. Within the resin tag $(\mathrm{T})$, an electron-dense line representing the lamina limitans could be seen (pointers). The surface electron-dense zone (Hs) was continuous with similar zones along the periphery of the tubular lumen (K). Lateral branches in cross-sections (black arrowheads) and a longitudinal section, branching out from a dentinal tubule (white arrow), could be identified. The presence of an electrondense zone around these lateral branches constituted the incomplete basal zone $(\mathrm{Hb})$. The subsurface "hybridoid region" $\left(\mathrm{H}^{\prime}\right)$ could not be readily distinguished from the underlying EDTA demineralized dentin matrix (D). $R$, resin layer. Bar $=2 \mu \mathrm{m}$. 


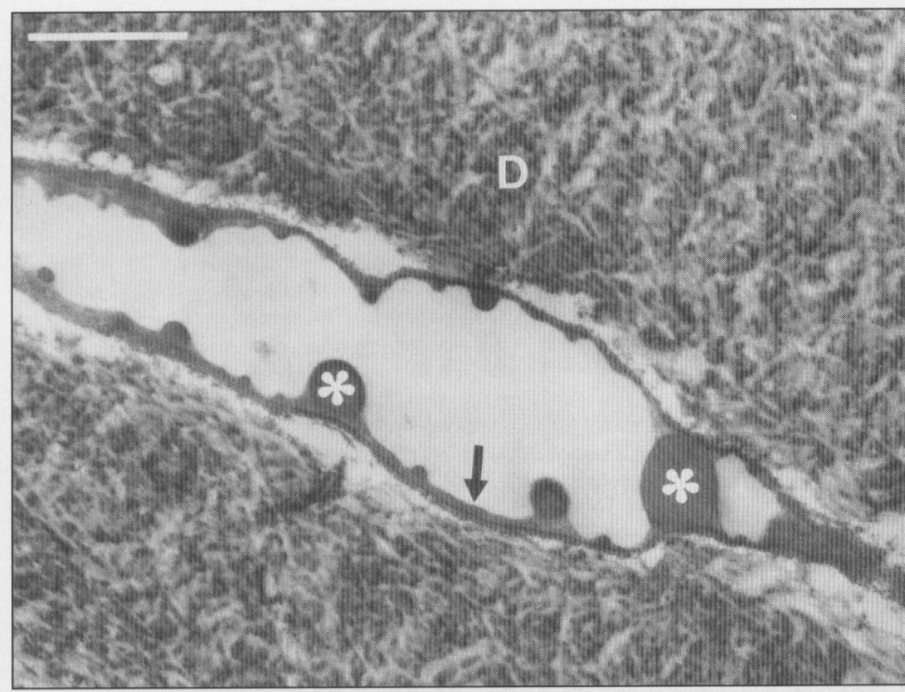

Figure 6a. Transmission electron micrograph of a dentinal tubule in the experimental formulation moist group, where resin globules (asterisks) were joined by a thin sheath of resin (arrow) to form a hollow resin tag. $\mathrm{D}$, intertubular dentinal matrix resulting from EDTA demineralization for TEM processing. Bar $=1 \mu \mathrm{m}$.

traversed by lateral branches from the dentinal tubules.

Pashley et al. (1993) distinguished between transdentinal permeation, i.e., the intratubular penetration of resin to form resin tags, and intradentinal permeation, i.e., the infiltration of resin into demineralized intertubular dentin. Dentinal tubule anastomosis through an interlocking network of resin-filled lateral branches has also been suggested as a potential contributing factor in micromechanical bonding to dentin (Chappell et al., 1994). It is clear from the results of our study that intratubular and intertubular permeation was adequate under moist-bonding conditions, resulting in a continous hybrid layer down to the demineralization front produced by the acid-dentin conditioners. Moreover, resin globules that were observed beyond short resin tags were similar to those previously reported in deep dentin in vivo (Tay et al., 1994, 1995d). Although effective intratubular permeation in the dry groups resulted in the formation of longer, solid resin tags within the dentinal tubules, vertical intertubular permeation was incomplete, resulting in a thin surface hybrid layer limited superficially to 1 to $2 \mu \mathrm{m}$. Circumferential intertubular permeation, being an extension of intratubular permeation through the periphery of the tubule lumina and their lateral branches, resulted in partial infiltration of the rest of the underlying demineralized dentin matrix. A thin hybrid layer was therefore observed along areas that were close to the course of the dentinal tubules and their lateral branches, leaving behind a subsurface, incompletely infiltrated "hybridoid region". Although remineralization of demineralized dentin collagen that retained its cross-banding (Kato and Fusayama, 1970; Miyauchi et al., 1978; Tatsumi, 1989) has been reported to be possible, its occurrence is doubtful in these isolated regions within the circumpulpal dentin, in the presence of resin tags in the dentinal tubules and with the possibility of damage to the odontoblast processes that could have limited the access to calcium and phosphate ions and phosphophoryns (Butler

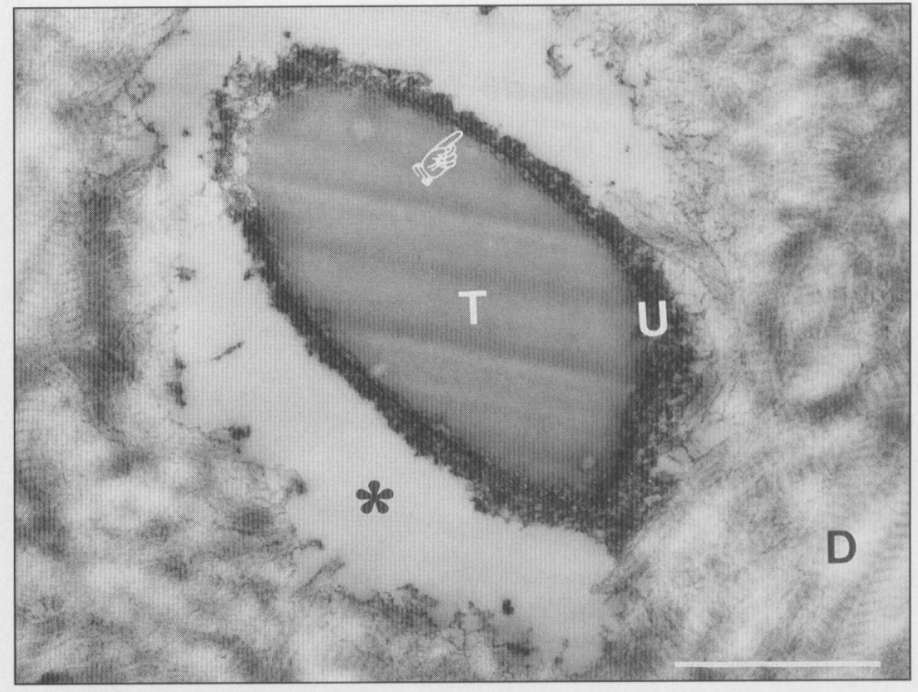

Figure $\mathbf{6 b}$. Transmission electron micrograph of a solid resin tag (T) in the experimental formulation dry group. The resin has diffused through the lamina limitans (pointer), trapping a layer of electron-dense material (U) within the peritubular space (asterisk). D, intertubular dentinal matrix that was demineralized by EDTA. Bar $=1 \mu \mathrm{m}$.

et al., 1992; Rabie and Veis, 1996). Furthermore, it is not known to what extent the resident noncollagenous proteins are extracted by different acids during the dentinconditioning step (Pashley et al., 1992), degraded in the absence of tissue turnover (Veis, 1993), or whether they are critical for the remineralization of demineralized dentin collagen (Takagi and Sasaki, 1986).

The bond between a restoration and tooth substrate might be equated with a chain, comprised of a series of links, which could only be as strong as its weakest link (Gwinnett, 1988). It is reasonable to assume that, in the dry groups, the weakest link within the resin-dentin interface resided within the hybrid layer (Kiyomura, 1987; Wang and Nakabayashi, 1991; Burrow et al., 1993; Kato et al., 1994). Pashley et al. (1992) and Sano et al. (1994) suggested that incomplete resin infiltration within the demineralized intertubular matrix resulted in a weak collagen-rich zone that is susceptible to hydrolysis and microleakage. On the other hand, properly infiltrated demineralized dentin (hybrid layer) has been shown to restore the ultimate tensile strength of mineralized dentin (Sano et al., 1995), an event predicted by the cohesive plateau theory (Adamson, 1982), with values greater than those reported for adhesion of resin to dentin. It is likely that the adhesive bond in the wet groups would fail along the interface between the adhesive resin and the top of the hybrid layer, as has been observed in previous studies (Tagami et al., 1993; Gwinnett and Yu, 1994; Tay et al., 1995b).

Ultrastructural features observed in both dry groups were comparable with results obtained in the application of the Clearfil Liner Bond system to dry, acid-conditioned dentin (Van Meerbeek et al., 1993), in which a similar electron-lucent zone could be observed between a surface and a basal electron-dense zone. In contrast, the hybrid layer observed in the moist groups in the present study was completely different, with no zonations and a notable 


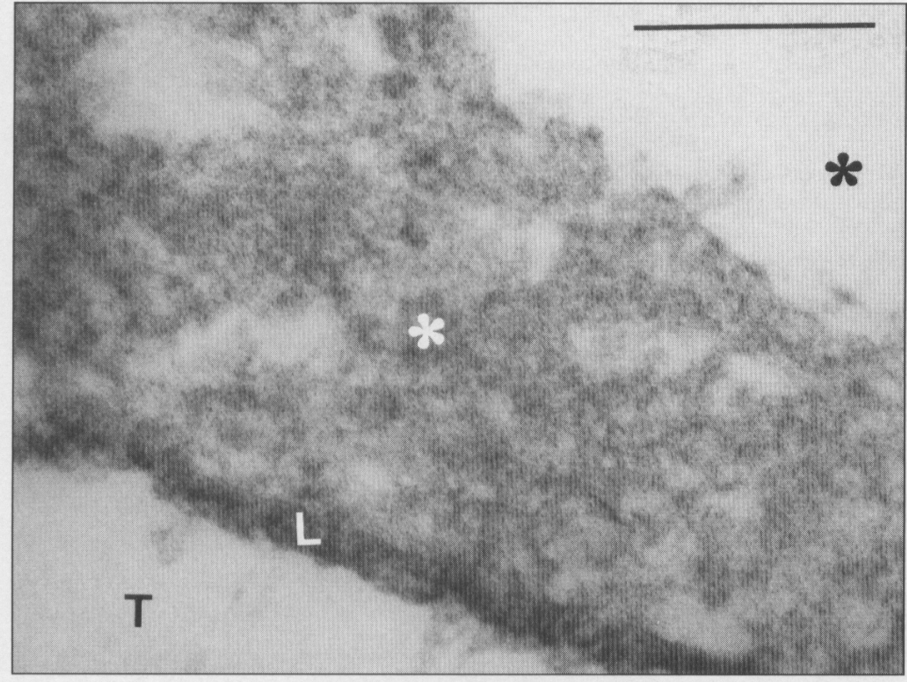

Figure 6c. Higher magnification of the electron-dense layer in Fig. 6b revealed the entrapment of a collection of amorphous material (white asterisk) by the resin following its diffusion through the lamina limitans (L) from the lumen of the dentinal tubule (T). Such a layer probably represented denatured proteins from the peritubular dentin. No resin-encapsulated hydroxyapatite crystallites could be observed. Black asterisk = peritubular space. Bar $=200 \mathrm{~nm}$.

absence of a basal electron-dense zone attributed to partially demineralized dentin. There appeared to be complete dentin demineralization with the $10 \%$ phosphoric acid, ending in a sharp demarcation between the zone of resin-infiltrated demineralized dentin and the underlying dentinal matrix which was subsequently demineralized and embedded in epoxy resin. This finding is in agreement with the work of Ikami et al. (1993). They also showed a thin layer of denatured collagen on the dentin surface but only after treatment with $38 \%$ phosphoric acid for $60 \mathrm{sec}$, an observation recently confirmed by Reeve et al. (1994). No denatured collagen was observed when dentin was conditioned with milder acids, an observation in common with the present study, where intact, banded collagen fibrils could be observed in moist groups right to the surface of the demineralized dentin.

If the dry group had been inspected without comparison with a moist group, it might be concluded that the electrondense surface zone in the dry groups was either a result of denaturation of surface collagen by the acid conditioner or denatured collagen remnants from the original smear layer. It might also be concluded that the electron-dense basal zone consisted of resin-infiltrated, partially demineralized hydroxyapatite crystallites. While one cannot refute the possibility of the occurrence of a zone of partially demineralized dentin with the use of some conditioners (Nakabayashi et al., 1992a, 1995; Van Meerbeek et al., 1993), it was evident from the TEM that such a zone was absent in the present study. The presence of electron-dense zones along the dentin surface, at the periphery of the dentinal tubules, as well as around their lateral branches suggested a similarity among these regions. They were all located adjacent to the liquid-vapor interface through which water has to pass during the process of evaporation. Water evaporating through and around the soft, demineralized collagen subjects it to crushing and tearing forces (Anderson, 1951) caused by the molecular linkage of water at the liquid-vapor boundary. The collagen fibrils protruding from the liquid surfaces could collapse and denature, resulting in a layer of amorphous material that restricted resin permeation (Pashley et al., 1993).

Similar to the problem encountered by Van Meerbeek et al. (1993), visualization of resin penetration into the decalcified dentin was difficult to achieve by TEM alone. However, when the moist and dry groups were compared, resin infiltration was more complete in the moist groups, where loosely arranged collagen fibers probably provided little resistance to the infiltration of the resin dissolved in either acetone or ethanol. At the same time, the acetone or ethanol displaced the water within the interfibrillar channels into which the resin infiltrated and formed a close physical relationship with the more loosely arranged tissue microstructure. In the dry groups, the electron-dense layers probably represented an amorphous mass of denatured collagen that, in the absence of interfibrillar spaces, acted as a molecular sieve, leading to a localized increase in resin concentration within the surface zone, while at the same time restricting diffusion of resin through this zone into the subsurface "hybridoid region". Such a molecular sieve effect may be similar to that proposed for resin infiltration of enamel (Darling et al., 1961).

It is speculated that water could be an essential component in some current adhesive systems, allowing desiccated collagen to be re-wetted prior to infiltration by bifunctional methacrylate components. Previous reports on the ability of HEMA to enhance the penetration capability of dentin substrates described the use of aqueous solutions of HEMA (Suzuki et al., 1990; Nakabayashi and Takarada, 1992; Nakabayashi et al., 1992b; Watanabe et al., 1993; Mizuno et al., 1994). Organic water-miscible solvents such as acetone, ethanol, and HEMA have been shown to exert a stiffening effect on demineralized dentin collagen (Pashley, 1996). Such chemical stabilization is useful when the collagen matrix is hydrated, allowing the integrity of the interfibrillar spaces to be maintained and facilitating resin infiltration. On the contrary, in the absence of water, chemical stiffening of a dehydrated and collapsed collagen matrix prevents its re-expansion and results in restricted resin infiltration. As illustrated in this study, application of "water-free primers" (i.e., primers with no additional water included in the solvent) to air-dried dentin resulted in incomplete hybridization. It might be argued that desiccating the acid-conditioned dentin for $30 \mathrm{sec}$ followed by the application of such "water-free primers" was far removed from the usual technique adopted in clinical practice, aggravating the results observed in the dry groups. However, by subjecting the specimens to such a degree of desiccation challenge, the authors wished to establish a paradigm for the behavior of these adhesives at one end of the spectrum - the overdry situation. There is probably a limit to the amount of moisture necessary for keeping the demineralized collagen stable so that the benefits of moist bonding can be optimized. Studies are currently being 
conducted in our laboratories on the reaction of these adhesives to various degrees of surface wetness. Application of "water-containing primers" (i.e., hydrophilic resins dissolved primarily in acetone but with different amounts of water added by the manufacturers as part of the solvent) to an already-moist dentin surface, for example, represents the other end of the spectrum, resulting in an "overwet phenomenon" (Gwinnett et al., 1996; Pang et al., 1995; Tay et al., 1995c; Wei et al., 1995). In the presence of excessive water, some of these resins appear to undergo phase separation of the hydrophobic components of the resin composition, resulting in resin globule formation.

\section{Acknowledgments}

This investigation was supported by the Committee on Research and Conference Grants (CRCG), the University of Hong Kong. The authors would like to thank Mr. Byoung Suh, President of Bisco, Inc., for generously formulating and providing the bottles of experimental primer used in this study. The technical expertise of Lee Man Tung and Simon Lee of the Oral Biology Unit, Prince Philip Dental Hospital, the University of Hong Kong, is also graciously acknowledged.

\section{References}

Abe Y (1986). Relation between interpenetration and bond strength to dentin with 4-META, phenyl P, HNPM, and 4-MET/MMA-TBB resins. J Jpn Dent Mater 5:839- 851.

Adamson AW (1982). Physical chemistry of surfaces. 4th ed. New York: John Wiley Publ., p. 18.

Anderson TF (1951). Techniques for the preservation of three-dimensional structure in preparing specimens for the electron microscope. Trans NY Acad Sci 13:130-133.

Berry EA, von der Lehr WN, Herrin HK (1987). Dentin smear layer treatments for the removal of the smear layer: an SEM study. J Am Dent Assoc 116:65-67.

Bowen RL (1978). Adhesive bonding of various materials to hard tooth tissues-solubility of dentinal smear layer in dilute acid buffers. Int Dent J 28:97-107.

Burrow MF, Tagami J, Hosoda H (1993). The long term durability of bond strengths to dentin. Bull Tokyo Med Dent Univ 40:173-191.

Butler WT, D'Souza RN, Bronckers AL, Happonen RP, Somerman MJ (1992). Recent investigations on dentin specific proteins. Proc Finn Dent Soc 88(Suppl 1):369-376.

Chappell RP, Cobb CM, Spencer P, Eick JD (1994). Dentinal tubule anastomosis: a potential factor in dentinal adhesive bonding? J Prosthet Dent 72:183-188.

Chiba M, Itoh K, Wakumoto S (1989). Effect of dentin cleansers on the bonding efficacy of dentin adhesive. Dent Mater 8:76-85.

Darling AI, Mortimer KV, Poole DFG, Ollis WD (1961). Molecular sieve behaviour of normal and carious enamel. Arch Oral Biol 5:251-273.

Eick JD, Cobb CM, Chappell RP, Spencer P, Robinson SJ (1991). The dentinal surface: its influence on dentinal adhesion. Part I. Quintessence Int 22:967-977.

Eick JD, Robinson SJ, Cobb CM, Chappell RP, Spencer P (1992).
The dentinal interface: Its influence on dentinal adhesion. Part II. Quintessence Int 23:43-51.

Eick JD, Robinson, SJ, Chappell RP, Cobb CM, Spencer P (1993). The dentinal surface: Its influence on dentinal adhesion. Part III. Quintessence Int 24:571-582.

Erickson RL (1992). Surface interactions of dentin adhesive materials. Oper Dent Suppl 5:81-94.

Garofalo S, Metsäranta M, Ellard J, Smith C, Horton W, Vuorio E, et al. (1993). Assembly of cartilage collagen fibrils is disrupted by overexpression of normal type II collagen in transgenic mice. Proc Natl Acad Sci 90:3825-3829.

Gwinnett AJ (1988). Bonding of restorative resins to enamel. Int Dent J 38:91-96.

Gwinnett AJ (1992). Moist versus dry dentin: its effect on shear bond strength. Am J Dent 5:127-129.

Gwinnett AJ (1994a). Dentin bond strength after air drying and rewetting. Am J Dent 7:144-148.

Gwinnett AJ (1994b). Chemically conditioned dentin: a comparison of conventional and environmental scanning electron microscopy findings. Dent Mater 10:150-155.

Gwinnett AJ, Kanca J III (1992a). Micromorphological relationship between resin and dentin in vivo and in vitro. Am J Dent 5:19-23.

Gwinnett AJ, Kanca JA III (1992b). Micromorphology of the bonded dentin interface and its relationship to bond strength. Am J Dent 5:73-77.

Gwinnett AJ, Yu S (1994). Effect of long-term water storage on dentin bonding. Am J Dent 8:109-111.

Gwinnett AJ, Tay FR, Pang KM, Wei SHY (1996). Bridging the gap between the overdry and overwet phenomenonoptimization of resin adhesion and dentinal seal. Proceedings of The International Conference on Dentin/Pulp Complex 1995. Quintessence Int (in press).

Hosoda H, Fujitani M, Negishi T, Hirasawa K (1989). Effect of a series of new cavity surface treatments on bond strength and wall adaptation of adhesive composite resins. Jpn J Conserv Dent 32:656-665.

Hunter KE, Duke ES, Conn LJ (1993). Interpenetration of resin into variable dentin substrate (abstract). J Dent Res 72(Spec Iss):136.

Ikami K, Fukushima M, Usami Y, Iwaku M (1993). The effects of surface conditioners on dentin structure (abstract). J Dent Res 72(Spec Iss):211.

Inokoshi S, Hosoda $\mathrm{H}$, Harniratissai C, Shimida $\mathrm{Y}$, Tatsumi T (1990). A study on the resin impregnated layer of dentin: Part I. A comparative study on the decalcified and undecalcified sections and the application of argon ion beam etching to disclose the resin impregnated layer of dentin. Jpn J Conserv Dent 33:427-442.

Inokoshi S, Hosoda H, Harniratissai C, Shimada Y (1993). Interfacial structure between dentin and seven dentin bonding systems revealed using argon ion beam etching. Oper Dent 18:8-16.

Kanca J III (1992). Improving bond strength through acid etching of dentin and bonding to wet dentin surfaces. $J \mathrm{Am}$ Dent Assoc 123:35-43.

Kato G, Watanabe I, Nakabayashi N (1994). Effect of 4-META acetone solution on bonding of resin to dentin etched with phosphoric acid. J Jpn Dent Mater 13:29-35. 
Kato S, Fusayama T (1970). Recalcification of artificially decalcified dentin in vivo. J Dent Res 49:1061-1067.

Kiyomura M (1987). Bonding strength to bovine dentin with 4-META/MMA-TBB resin-long term stability and influence of water. J Jpn Dent Mater 6:860-872.

Kramer IR, McLean JW (1952). Alterations in the staining reactions of dentine resulting from a constituent of a new self-polymerising resin. Br Dent J 93:150-153.

Lee HL Jr, Orlowski JA, Scheidt GC, Lee JR (1973). Effects of acid etchants on dentin. J Dent Res 52:1228-1233.

Luft JH (1971). Ruthenium red and violet. II. Fine structural localization in animal tissues. Anat Rec 171:369-415.

Miller RG, Bowles CQ, Gutshall PL, Eick JD (1994). The effects of ion sputtering on dentin and its relation to depth profiling. J Dent Res 73:1457-1461.

Miyauchi H, Iwaku M, Fusayama T (1978). Physiological recalcification of carious dentin. Bull Tokyo Med Dent Univ 25:169-179.

Mizuno Y, Suzuki K, Kondo Y, Yatani H, Nakai H, Yamashita A (1994). Adhesiveness of resin to acid-etched dentin. Part 2. Structural change of adherent surface and adhesiveness, following to the pretreatment with solution of HEMA. J Jpn Dent Mater 13:475-482.

Mizunuma T (1986). Relationship between bond strength of resin to dentin and structural change of dentin collagen during etching. Influence of ferric chloride to structure of the collagen. J Jpn Dent Mater 5:54-64.

Nakabayashi N (1982). Resin reinforced dentin due to infiltration of monomers into dentin at the adhesive interface. J Jpn Dent Mater 1:78-81.

Nakabayashi N (1992). Adhesive bonding with 4-META. Oper Dent Suppl 5:125-130.

Nakabayashi N, Takarada K (1992). Effect of HEMA on bonding to dentin. Dent Mater 8:125-130.

Nakabayashi N, Kojima K, Masuhara E (1982). The promotion of adhesion by the infiltration of monomers into tooth substrates. J Biomed Mater Res 16:265-273.

Nakabayashi N, Ashizawa M, Nakamura M (1992a). Identification of a resin-dentin hybrid layer in vital human dentin created in vivo: durable bonding to vital dentin. Quintessence Int 23:135-141.

Nakabayashi N, Watanabe A, Gendusa NJ (1992b). Dentin adhesion of "modified" 4- META/MMA-TBB resin: function of HEMA. Dent Mater 8:259-264.

Nakabayashi N, Watanabe A, Ikeda W (1995). Intra-oral bonding of 4-META/MMA-TBB resin to vital human dentin. Am J Dent 8:37-42.

Pang KM, Tay FR, Gwinnett AJ, Wei SHY (1995). Phase separation of All-Bond 2 primers with excess surface moisture (abstract). J Dent Res 74(Spec Iss):403.

Pashley DH (1996). The effect of dentin bonding procedures on the dentin/pulp complex. Proceedings of The International Conference on Dentin/Pulp Complex 1995. Quintessence Int (in press).

Pashley DH, Horner JA, Brewer PD (1992). Interactions of conditioners on the dentin surface. Oper Dent Suppl 5:137-150.

Pashley DH, Ciucchi BC, Sano H, Horner JA (1993). Permeability of dentin to adhesive agents. Quintessence Int 24:618-631.
Perdigao J, Swift EJ Jr (1994). Adhesion of a total-etch phosphate ester bonding agent. Am J Dent 7:149-152.

Rabie AM, Veis A (1996). An immunocytochemical study of the routes of secretion of collagen and phosphophoryn from odontoblasts into dentin. Connect Tissue Res (in press).

Reeve JB, Hunter KE, Conn LJ, Duke ES (1994). Phosphoric acid concentration and time on the resin interdiffusion (abstract). J Dent Res 73(Spec Iss):223.

Ruse ND, Smith DC (1991). Adhesion to bovine dentin-surface characterization. J Dent Res 70:1002-1008.

Sano H, Shono T, Takatsu T, Hosoda H (1994). Microporous dentin zone beneath resin-impregnated layer. Oper Dent 19:59-64.

Sano H, Takatsu T, Ciucchi B, Russell CM, Pashley DH (1995). Tensile properties of resin-infiltrated demineralized human dentin. J Dent Res 74:1093-1102.

Sugizaki J (1991). The effect of various primers on the dentin adhesion of resin composites-SEM and TEM observations of the resin impregnated layer and adhesion promoting effect of the primers. Ipn J Conserv Dent 34:228-265.

Suzuki K, Takahashi M, Nakai H (1990). Treatment of dentin by aqueous solution of amino acid derivatives-HEMA. Adhesive Dent 8:43-51.

Tagami J, Nakajima M, Shono T, Takatsu T, Hosoda H (1993). Effect of aging on dentin bonding. Am J Dent 6:145-147.

Takagi Y, Sasaki S (1986). Histological distribution of phosphophoryn in normal and pathological human dentins. J Oral Pathol 15:463-467.

Takarada K (1990). Stable adhesion to dentin. Combination of EDTA 3-2 (NH4/Fe) pretreatment and 2\% 4-META/MMATBB resin. J Jpn Dent Mater 9:841-849.

Tam LE, Pilliar RM (1994). Fracture surface characterization of dentin-bonded interfacial fracture toughness specimens. I Dent Res 73:607-619.

Tatsumi T (1989). Physiological remineralization of artificially decalcified monkey dentin under adhesive composite resin restoration. J Jpn Stomatol Soc 56:49-74.

Tay FR, Gwinnett AJ, Pang KM, Wei SHY (1994). Structural evidence of a sealed tissue interface with a total-etch wet-bonding technique in vivo. J Dent Res 73:629-636.

Tay FR, Gwinnett AJ, Pang KM, Wei SHY (1995a). Micromorphologic relationship of the resin dentin interface following a total etch technique in vivo using a dentinal bonding agent. Quintessence Int 26:63-70.

Tay FR, Gwinnett AJ, Pang KM, Wei SHY (1995b). Variability in microleakage observed in a total etch wet bonding technique under different handling conditions. J Dent Res 74:1168-1178.

Tay FR, Gwinnett AJ, Pang KM, Wei SHY (1995c). “The overwet phenomenon": A TEM study on possible mechanisms (abstract). J Dent Res 74(Spec Iss):402.

Tay FR, Pang KM, Gwinnett AJ, Wei SHY (1995d). A scanning electron microscopic study of the extent of resin penetration into human coronal dentin following a total etch technique in vivo. Cells and Mater 4:317-329.

Titley K, Chernecky R, Maric B, Valiquette N, Smith D (1994). The morphology of the demineralized layer in primed dentin. Am J Dent 7:22-26.

Van Meerbeek B, Inokoshi S, Braem M, Lambrechts P, Vanherle 
G (1992). Morphological aspects of the resin-dentin interdiffusion zone with different dentin adhesive systems. J Dent Res 71:1530-1540.

Van Meerbeek B, Dhem A, Goret-Nicaise M, Braem M, Lambrechts P, Vanherle G (1993). Comparative SEM and TEM examination of the ultrastructure of the resin-dentin interdiffusion zone. J Dent Res 72:495-501.

Veis A (1993). Mineral-matrix interactions in bone and dentin. J Bone Miner Res 8(Suppl 2):493-497.

Walshaw PR, McComb D (1994). SEM evaluation of the resindentin interface with proprietary bonding agents in human subjects. J Dent Res 73:1079-1087.
Wang T, Nakabayashi N (1991). Effect of 2-(methacryloxy)-ethyl phenyl hydrogen phosphate on adhesion to dentin. J Dent Res 70:59-66.

Watanabe I, Hiranuma K, Nakabayashi N (1993). Effect of HEMA on bonding to dentin pretreated with phosphoric acid. J Jpn Dent Mater 12:705-713.

Watanabe I, Nakabayashi N, Pashley DH (1994). Bonding to ground dentin by a phenyl-P self-etching primer. J Dent Res 73:1212-1220.

Wei SHY, Tay FR, Gwinnett AJ, Pang KM (1995). "The overwet phenomenon": A SEM study of surface resin globule formation (abstract). J Dent Res 74(Spec Iss):402. 